\title{
Degradation of different pectins by fungi: correlations and contrasts between the pectinolytic enzyme sets identified in genomes and the growth on pectins of different origin
}

\author{
Isabelle Benoit ${ }^{1 *}$, Pedro M Coutinho ${ }^{2}$, Henk A Schols ${ }^{3}$, Jan P Gerlach ${ }^{1}$, Bernard Henrissat ${ }^{2}$ and Ronald P de Vries ${ }^{1,4}$
}

\begin{abstract}
Background: Pectins are diverse and very complex biomolecules and their structure depends on the plant species and tissue. It was previously shown that derivatives of pectic polymers and oligosaccharides from pectins have positive effects on human health. To obtain specific pectic oligosaccharides, highly defined enzymatic mixes are required. Filamentous fungi are specialized in plant cell wall degradation and some produce a broad range of pectinases. They may therefore shed light on the enzyme mixes needed for partial hydrolysis.

Results: The growth profiles of 12 fungi on four pectins and four structural elements of pectins show that the presence/absence of pectinolytic genes in the fungal genome clearly correlates with their ability to degrade pectins. However, this correlation is less clear when we zoom in to the pectic structural elements.

Conclusions: This study highlights the complexity of the mechanisms involved in fungal degradation of complex carbon sources such as pectins. Mining genomes and comparative genomics are promising first steps towards the production of specific pectinolytic fractions.
\end{abstract}

\section{Background}

Pectin is one of the major and one of the most complex plant cell wall components [1]. It forms a family of diverse polysaccharides composed of 17 different monomers containing more than 20 different glycosidic linkages [2,3]. Pectin is composed of six covalently linked substructures that are arranged to form a backbone substituted with side chains. Three pectic structural elements (homogalacturonan, rhamnogalacturonan-I, and substituted galacturonans) have been isolated from primary cell walls and were structurally characterized $[4,5]$, (Figure 1, [6]). The architecture of pectin is dependent on the plant species and tissue, resulting in strong variations in the ratio of these different polysaccharides as well as their level of substitution $[7,8]$. The possible cross links between the different polysaccharide constituents are still not fully elucidated and several different models have been proposed to

\footnotetext{
*Correspondence: benoit@uu.nl

${ }^{1}$ Microbiology \& Kluyver Centre for Genomics of Industrial Fermentations, Utrecht University, Padualaan 8, Utrecht 3584 CH, The Netherlands Full list of author information is available at the end of the article
}

describe pectin architecture. In addition, some proteins are known to be associated to pectin, such as extensin in sugar beet pectin [9] and arabinogalactan proteins [10].

Pectin is an important and versatile additive in several food types because of its hydrocolloid character that makes it a gelling, stabilizing or thickening agent. The emulsification properties of sugar beet pectin due to a high content of acetyl groups and proteins make it as an interesting component for microencapsulation [11].

As a dietary fibre naturally present in plant products, it also provides nutritional benefits for the human diet. Derivatives of pectic polymers and oligosaccharides from pectin were shown to have positive effects on human health. These included immuno-regulatory effects in the intestine, lowering the blood cholesterol level, and slowing down the absorption of glucose in the serum of diabetic and obese patients [12,13]. A modified form of citrus pectin appears to be effective for a range of cancers at all stages of development and was patented as anti-cancer agent $[14,15]$. Moreover, pectin oligosaccharides proved to be good probiotic compounds [16] [17]. 


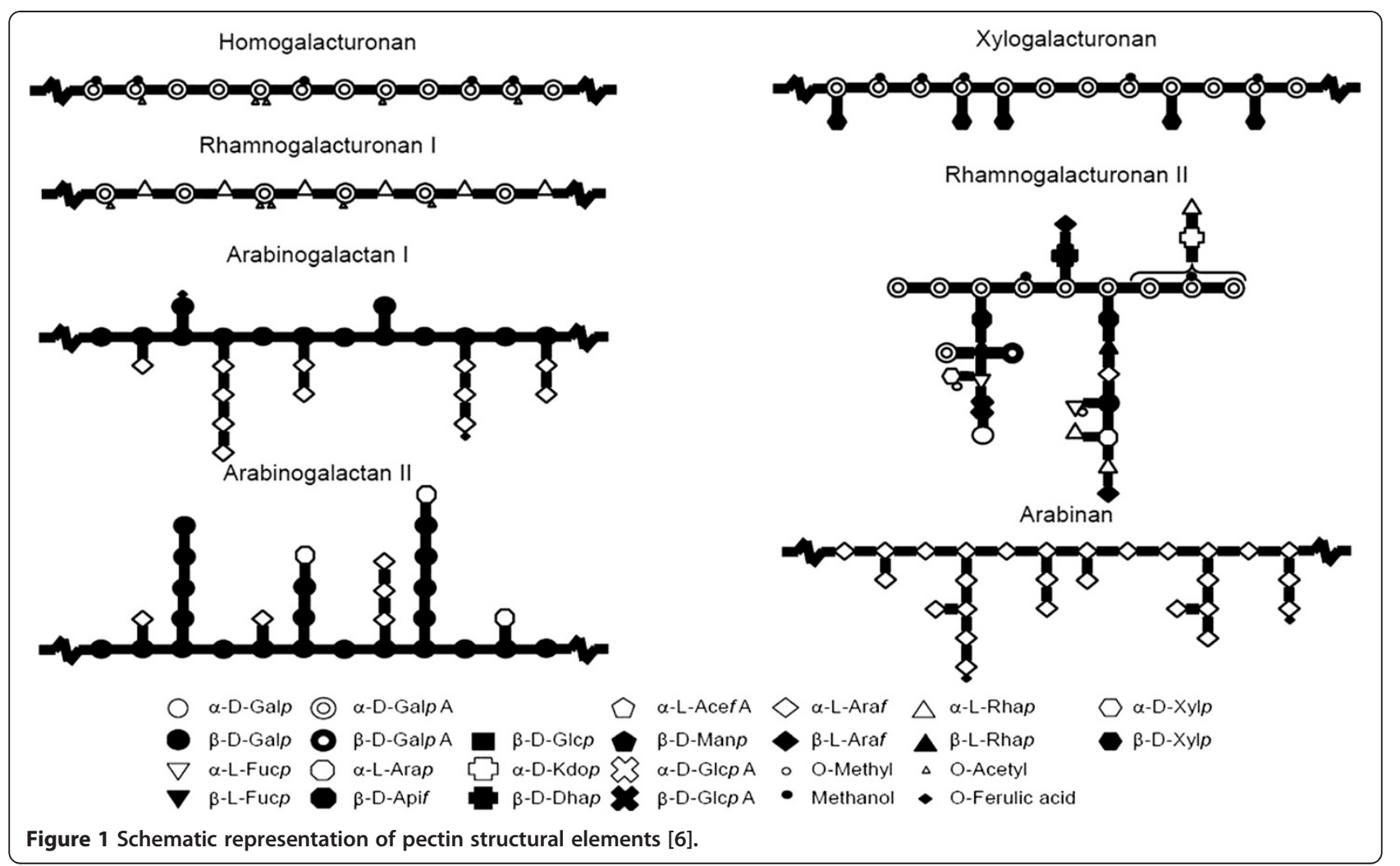

Enzymatic hydrolysis of pectin has advantages over chemical hydrolysis as enzymes target specific linkages of the pectin molecules while chemical methods are less specific $[18,19]$. This is especially important when partial hydrolysis is required to produce specific fragments. To meet the demand for specific pectin-derived oligosaccharides, specialized mixtures of pectinolytic enzymes are required that cleave only those linkages necessary to produce these oligosaccharides, but do not further hydrolyse them.

Most of the current commercial pectinolytic enzyme mixtures are produced by filamentous fungi. These organisms are very efficient in the degradation of plant cell wall polysaccharides and use a broad set of enzymes to convert them into monomeric sugars that can be taken up as nutrients. However, the composition of these enzyme sets differs significantly between fungal species and this is also observed for the subset of pectinolytic enzymes. For instance Rhizopus spp. mainly degrades the homogalacturonan part of pectin, while Aspergilli produce enzymes to hydrolyse all pectic structural elements [20]. A better understanding of the correlation between the make-up of these enzyme sets and hydrolysis of different structural elements of pectin is likely to result in novel strategies to produce tailor-made pectinase preparations for the production of specific pectin-oligosaccharides.

The goal of this study was to compare the pectinolytic potential (based on their genome) of twelve fungi and their ability to use pectin (components) as a carbon source. These fungi were chosen because of their different life-styles and natural habitats. Some of these fungi have been studied in detail in relation to pectin degradation, while in others this topic is largely untouched. Four are ascomycete plant pathogens of which one is specific for cereals (Magnaporthe oryzae), while the other have a broad host plant range (Giberella zeae, Botrytis fuckeliana, Sclerotinia sclerotiorum) while the other eight are saprobes. The saprobic ascomycetes include model organisms (Aspergillus nidulans, Trichoderma virens, Podospora anserina) and industrial fungi that are commonly used for enzyme production (Aspergillus niger, Aspergillus oryzae). The basidiomycete Phanerochaete chrysosporium has been the most intensively studied white rot fungus because of its powerful peroxidases and oxidases that are involved in lignin degradation [21], but little is known about its pectinolytic potential. The zygomycete Rhizopus oryzae is used in industrial fermentations for the production of lactic acid [22] and ethanol. It was found that the production rates of those two metabolites were affected by the activity of the pectinolytic enzymes $[23,24]$. The ascomycete Aspergillus clavatus is mainly studied in relation to the production of secondary metabolites $[25,26]$ and the toxin patulin which may be associated with disease in humans and animals [27].

All fungi were grown on pectins from four different origins (Table 1) as well as on four structural elements 
of pectins: arabinan, galactan, rhamnogalacturonan I (RGI) and homogalacturonan (PGA). The ability to use the pectins and their structural elements as carbon source was compared to the pectinolytic potential of the fungi that was derived from their genome sequence.

\section{Results and discussion}

Genome annotation reveals significant differences in the pectinolytic potential of the twelve fungi

The Carbohydrate-active enzymes (CAZymes) related to pectin degradation of the twelve fungi tested in this study were identified using the CAZy annotation pipeline [28] and compared to each other (Table 2). Six of these fungi had a high number of CAZymes involved in pectin degradation while the other six had a lower content of these CAZymes. For instance $P$. anserina has 12 glycoside hydrolases, 7 polysaccharide lyases and 2 carbohydrate esterases involved in pectin degradation, while $A$. oryzae has 52 glycoside hydrolases (GH), 20 polysaccharide lyases (PL) and 9 carbohydrate esterases (CE) (Table 2). The CAZy annotations are based upon the protein models derived from the annotation of the genomes. As the pipelines from JGI and Broad are not identical in the identification of protein models, this may cause some differences in the numbers per family which may have had a small effect on the comparison. The CAZy families involved in pectin degradation and the activities that can be found within these families are listed in Table 3. Family PL11 (rhamnogalacturonan lyases) was not taken into account in the remainder of this study since only $A$. nidulans has one putative enzyme of this family which has not been biochemically characterised.

Distinct growth profiles of the twelve fungi on pectins and pectin structural elements can be in part explained by differences in CAZy content of their genome

Growth on pectins from soy, apple, citrus and sugar beet and on four structural elements of pectin demonstrated different profiles for the twelve fungi of this study
(Additional file 3). As the different species have different growth rates, glucose was used as standard for growth. Growth on the pectins and pectin structural elements was compared to growth on glucose and this relative growth difference was then used to compare the species to each other. $A$. niger, $A$. oryzae, $A$. nidulans and $B$. cinerea grew very well on pectin, while $A$. clavatus, $P$. anserina and $P$. chrysosporium grew poorly (Figure 2). The analysis of the CAZy families involved in pectin degradation in these species showed that the relative number of pectinases is lower for the species that do not grow well than for the species that grow well. P. chysosporium, A. clavatus $P$. anserina have 3,2 and no GH28 members, respectively, while $A$. oryzae, $A$. niger and $A$. nidulans have respectively 20,21 and 10 GH28 members with at least one gene encoding each activity of this family (Table 2). A clavatus, P. chrysosporium and P. anserina are also poorly equipped with families PL1, PL3, PL4 and PL9 when compared to the four other species.

These results show that the growth profiles on pectins and the overall pectinolytic enzyme content correlate. A similar correlation was observed in studies concerning xylan or cellulose degradation [29,30].

However, this correlation is less obvious when we compare growth on the structural elements of pectin to the presence of specific subsets of pectinases. For example, soy pectin does not contain homogalacturonan but a high proportion of rhamnogalacturonan I (Table 1). However, fungi that grow well on this pectin such as A. nidulans, G. zeae and $P$ anserina do not always have an increased rhamnogalacturonan degrading potential (families GH28 RHG, RGX; GH51; GH54; GH93; GH43, ABN; PL1; PL3; CE8) compared to their homogalacturonan degrading potential (families GH28 PGA, PGX; PL4 and CE12). Interestingly, this also applies to $A$. clavatus, which, despite its overall poor growth, grows better on soy pectin than on the other substrates. And the fungi that have the higher rhamnogalacturonan degrading potential do not grow better on RGI. The best growth measured on RGI is for T. virens which has less rhamnogalacturonan degrading enzymes (only 10) than the

Table 1 Sugar composition ( $\mathrm{mol} \%$ ), acetylation and methylesterification degrees of the pectic polysaccharides used in this study

\begin{tabular}{|c|c|c|c|c|c|c|c|c|c|c|}
\hline & Fuc & Rha & Ara & Gal & Glc & Xyl & Uronic acids & $\mathrm{DA}^{*}$ & $\mathrm{DM}^{*}$ & reference \\
\hline Soy pectin & 4 & 5 & 25 & 39 & 3 & 7 & 17 & 23 & 68 & This study \\
\hline Sugar beet Beta & - & 5 & 7 & 11 & 0.4 & 1 & 73 & 14 & 30 & This study \\
\hline Lemon pectin & - & 2 & 3 & 6 & 1 & 0.2 & 88 & - & 70 & This study \\
\hline Apple pectin & - & 3 & 3 & 8 & 2 & 2 & 82 & - & 72 & This study \\
\hline Galactan (potato) & - & 3 & 3 & 88 & - & - & 6 & - & - & Megazyme \\
\hline Arabinan (sugar beet) & - & 2 & 88 & 3 & - & - & 7 & - & - & Megazyme \\
\hline RGI (potato) & - & 20 & 3.3 & 12 & - & 1 & 62 & - & - & Megazyme \\
\hline PGA (citrus) & - & - & - & - & - & - & $>97$ & - & - & Sigma \\
\hline
\end{tabular}

*Moles of acetyl (DA) groups or methyl (DM) esters groups per 100 moles of GalA. 
Table 2 Pectinolytic glycoside hydrolases, polysaccharide lyases and carbohydrate esterases of the 12 fungal species used in this study

\begin{tabular}{|c|c|c|c|c|c|c|c|c|c|c|c|c|}
\hline $\begin{array}{l}\text { CAZy } \\
\text { family }\end{array}$ & $\begin{array}{c}\text { S. } \\
\text { sclerotiorum }\end{array}$ & $\begin{array}{c}\text { B. } \\
\text { fuckeliana }\end{array}$ & $\begin{array}{c}A . \\
\text { nidulans }\end{array}$ & $\begin{array}{c}\text { A. } \\
\text { clavatus }\end{array}$ & $\begin{array}{c}\text { A. } \\
\text { oryzae }\end{array}$ & $\begin{array}{c}\text { A. } \\
\text { niger }\end{array}$ & $\begin{array}{c}M . \\
\text { orysae }\end{array}$ & $\begin{array}{c}P . \\
\text { anserina }\end{array}$ & $\begin{array}{c}T . \\
\text { virens }\end{array}$ & $\begin{array}{c}G . \\
\text { zeae }\end{array}$ & $\begin{array}{c}P . \\
\text { chrysosporium } \\
\end{array}$ & $\begin{array}{c}R . \\
\text { oryzae }\end{array}$ \\
\hline$\overline{\mathrm{GH} 2} \mathrm{LAC}$ & 1 & 1 & 3 & 0 & 1 & 0 & 3 & 3 & 1 & 3 & 0 & 0 \\
\hline GH28 (Total) & 16 & 18 & 10 & 2 & 20 & 21 & 3 & 0 & 6 & 6 & 3 & 18 \\
\hline - PGA & 6 & 9 & 3 & 1 & 5 & 7 & 1 & 0 & 2 & 2 & 1 & 15 \\
\hline - PGX & 2 & 2 & 3 & 1 & 2 & 5 & 1 & 0 & 2 & 3 & 1 & 3 \\
\hline$-\mathrm{RHG}$ & 4 & 4 & 1 & 0 & 6 & 6 & 1 & 0 & 0 & 1 & 1 & 0 \\
\hline - RGX & 3 & 2 & 2 & 0 & 5 & 2 & 0 & 0 & 2 & 0 & 0 & 0 \\
\hline$-X G H$ & 1 & 1 & 1 & 0 & 2 & 1 & 0 & 0 & 0 & 0 & 0 & 0 \\
\hline GH35 LAC & 4 & 4 & 4 & 3 & 7 & 5 & 0 & 1 & 1 & 3 & 3 & 1 \\
\hline GH43 (Total)* & 4 & 5 & 15 & 13 & 20 & 11 & 20 & 10 & 3 & 17 & 4 & 2 \\
\hline$-A B F$ & 0 & 0 & 3 & 0 & 3 & 0 & 6 & 2 & 0 & 0 & 0 & 0 \\
\hline - ABN & 1 & 1 & 4 & 5 & 5 & 4 & 1 & 0 & 0 & 1 & 1 & 2 \\
\hline GH78 RHA & 4 & 8 & 9 & 0 & 8 & 8 & 3 & 1 & 3 & 7 & 0 & 0 \\
\hline GH88 & 0 & 1 & 3 & 0 & 3 & 1 & 1 & 0 & 3 & 1 & 1 & 0 \\
\hline GH93 ABX & 1 & 1 & 2 & 1 & 3 & 1 & 1 & 3 & 1 & 2 & 0 & 0 \\
\hline GH105 URGH & 1 & 1 & 4 & 3 & 2 & 2 & 3 & 0 & 1 & 3 & 0 & 0 \\
\hline GH51 ABF & 2 & 3 & 3 & 3 & 3 & 4 & 3 & 1 & 0 & 2 & 2 & 0 \\
\hline GH53 GAL & 2 & 2 & 1 & 0 & 1 & 1 & 1 & 1 & 0 & 1 & 1 & 0 \\
\hline GH54 ABF & 1 & 1 & 1 & 1 & 1 & 1 & 1 & 0 & 2 & 1 & 0 & 0 \\
\hline GH127 ABF & 0 & 1 & 1 & 1 & 0 & 0 & 0 & 0 & 0 & 1 & 0 & 0 \\
\hline PL1 PEL, PLY & 4 & 6 & 9 & 2 & 12 & 7 & 2 & 4 & 0 & 9 & 0 & 0 \\
\hline PL3 PLY & 0 & 2 & 5 & 1 & 3 & 0 & 1 & 2 & 0 & 7 & 0 & 0 \\
\hline PL4 RGL & 0 & 0 & 4 & 2 & 4 & 2 & 1 & 1 & 0 & 3 & 0 & 0 \\
\hline PL9 PLY & 0 & 0 & 1 & 0 & 1 & 0 & 0 & 0 & 0 & 1 & 0 & 0 \\
\hline CE8 PME & 5 & 5 & 3 & 7 & 5 & 3 & 1 & 1 & 2 & 6 & 0 & 6 \\
\hline CE12 RGAE & 3 & 3 & 2 & 1 & 4 & 2 & 3 & 1 & 0 & 3 & 0 & 0 \\
\hline
\end{tabular}

* Total refers to all the GH43 including the activities not directly involved in pectin degradation such as $\beta$-xylosidase or exo-b-1,3-galactanase. Additional file 2 .

other fungi of this study (18 in average). However, 10 enzymes are probably sufficient to degrade rhamnogalacturonan. More genes coding for the same putative activity could mean more possibilities/options to achieve an efficient degradation. So far, the regulation of the expression of the pectinolytic activities is not fully understood and is likely a determining factor for the production level of pectin related enzymes. For instance, most fungi that grow on cellulose produce a wide range of cellulases. Nonetheless, the best studied cellulolytic fungus, Trichoderma reesei, only produces a small number at very high levels [31].

In order to visualize better the growth profiles and compare them to each other, a double hierarchical clustering was calculated from the growth profile of the 12 fungi on the four pectins and four pectic structural elements (Figure 3). The fungal species clustered in three main groups. The largest group contains $B$. cinerea, $P$. chrysosporium, A. niger, G. zeae, R. oryzae, S. sclerotiorum and $A$. oryzae. The second group is made of $A$. nidulans, $A$. clavatus and $P$. anserina and the third group contains $M$. oryzae and $T$. virens. The substrates clustered in 2 main groups plus two separated branches. The first cluster contains polygalacturonic acid, arabinan and galactan, while the second cluster contains sugar beet, lemon and apple pectin. The two separated branches are soy pectin and potato rhamnogalacturonan I. The correlation between growth and genome content is clear at a two-dimension level, in that a high number of pectinases results in good growth rates. The complexity of the mechanisms involved appears when growth on pectin structural elements is compared to the presence of specific related activities.

In the paragraphs below, specific correlations will be discussed in more detail.

\section{Rhizopus oryzae is restricted to degradation of} unbranched pectins

The CAZy annotation of $R$. oryzae identified 18 putative polygalacturonases (GH28), one putative $\beta$-galactosidase 


\begin{tabular}{ll} 
Table 3 Activities of pectinolytic enzymes within CAZy \\
families & \\
\hline Glycoside hydrolases & \\
\hline GH2 & $\beta$-galactosidase \\
GH28 & \\
PGA & Endopolygalacturonase \\
PGX & Exopolygalacturonase \\
RHG & Endorhamnogalacturonase \\
RGX & Exorhamnogalacturonase \\
XGH & Xylogalacturonase \\
GH35 & $\beta$-galactosidase \\
GH43 & a-L-arabinofuranosidase, \\
endo-a-1,5-L-arabinanase \\
GH51 & a-L-arabinofuranosidase/endoglucanase \\
GH53 & endo-1,4- $\beta$-galactanase \\
GH54 & a-L-arabinofuranosidase/ $\beta$-xylosidase \\
GH78 & a-L-rhamnosidase \\
GH88 & D-4,5 unsaturated $\beta$-glucuronyl hydrolase \\
GH93 & exo-a-L-1,5-arabinanase \\
GH105 & unsaturated rhamnogalacturonyl hydrolase \\
GH127 & a-L-arabinofuranosidase \\
Polysaccharide lyases & \\
PL1 & pectate lyase/exo-pectate lyase/pectin lyase \\
PL3 & pectate lyase \\
PL4 & rhamnogalacturonan lyase \\
PL9 & pectate lyase/exopolygalacturonate lyase \\
PL11 & rhamnogalacturonan lyase \\
Carbohydrate esterases & \\
CE8 & \\
CE12 & \\
& pectin methylesterase \\
&
\end{tabular}

(GH35), 2 putative endo-arabinanases (GH43) and 6 putative pectin methyl-esterases (CE8). The $R$. oryzae genome contains a higher number of CAZymes related to pectin degradation than to the degradation of any other plant polysaccharide. Although it still has a rather small set of enzymes, this appears to be sufficient to (partially) degrade all four pectins to efficiently liberate carbon sources as good growth was observed on each of them. In a previous study, 3 of the 18 putative polygalacturonases were biochemically characterized as exo-polygalacturonases [24]. Both endo- and exo-polygalacturonases act on the homogalacturonan chains of the "smooth" region of pectin.

Interestingly, growth of $R$. oryzae was less good on the pectin structural elements. These results confirm the hypothesis that $R$. oryzae is restricted to the degradation of unbranched pectins. The analysis of Battaglia et al. [20] has shown that the enrichment of main-chain degrading enzymes of pectin in the $R$. oryzae genome was accompanied by a low number of accessory enzymes. This correlates with its life style as $R$. oryzae rot acts on PCW middle lamella of fruits and vegetables, which are rich in homogalacturonan (unbranched pectins).

On all substrates tested in this study, the growth profile of $R$. oryzae is similar to the growth profile of $S$. sclerotiorum that contains $16 \mathrm{GH} 28$ with at least one gene of each subfamily (Figure 3). This indicates that not all subfamilies of GH28 are essential to degrade pectin and liberate sufficient amounts of monomers to enable growth.

$R$. oryzae and S. sclerotiorum cluster based on their growth profile with G. zeae and A. oryzae (Figure 3). These four fungi have 5, 6, 6 and 5 pectin methyl esterases (PME, CE8) respectively. Two other fungi have several CE8 members: A. clavatus (7) and B. cinerea (5). These enzymes are responsible for removing methylesters from pectin. Different sensitivities of fungal endopolygalacturonases to methyl esterification have been reported [32,33]. The methyl esterification degree of the four pectins of our study differs significantly, with $30 \%$ for sugar beet, $68 \%$ for soy, $70 \%$ for lemon and $72 \%$ for apple pectin. A high number of pectin methyl esterases could be an advantage to release galacturonic acid that can be directly metabolized in combination with polygalacturonases that act mainly on pectins with a low degree of methyl esterification. The phylogenetic tree of GH28 (Additional file 1) demonstrates that the 18 polygalacturonases from $R$. oryzae cluster together but separately from the polygalacturonases of the other fungi, including endo-polygalacturonases $\mathrm{A}, \mathrm{B}, \mathrm{I}$ and II from $A$. niger (PgaA, PgaB, PgaI, PgaII). PgaA and PgaB are more active on methylesterified pectin and are clustered together with PgaI and PgaII that prefer nonmethylesterified pectin [32]. Although it can therefore not be concluded from the phylogenic tree, the high number of PMEs could indicate that $R$. oryzae polygalacturonases have higher affinity for non-methylated pectin.

\section{Magnaporte oryzae contains a small but complete set of pectinases}

In contrast to the highly specialised enzyme set from $R$. oryzae, $M$. oryzae appears to possess nearly all required activities albeit at low numbers. It has at least one gene coding for 15 pectinolytic activities out of the 17 activities we analysed (Table 3). The two missing activities are exorhamnogalacturonase and xylogalacturonase. The CAZy families required to degrade galactan are $\beta$ galactosidase (GH35) and $\beta$-endogalactanase (GH53). $M$. oryzae has no characterized GH35 $\beta$-galactosidases and has only one putative $\beta$-endogalactanase, nevertheless grew equally well on galactan as on arabinan, lemon pectin and apple pectin. M. oryzae has also $8 \mathrm{GH} 2$ members, but only three of those are candidate $\beta$-galactosidases and one is distantly related to $\beta$-galactosidases. These 


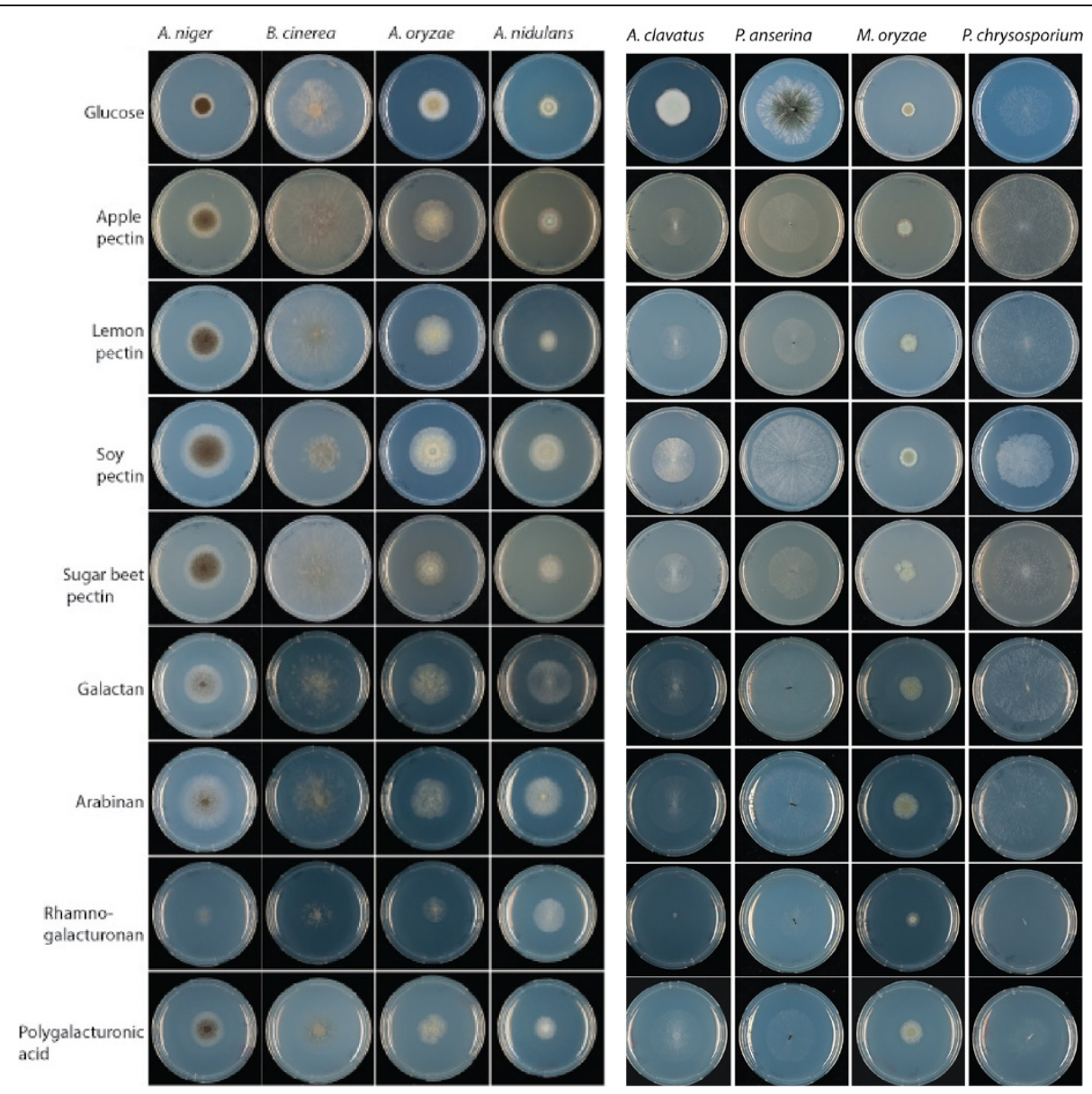

Figure 2 Correlation between growth profile and genome contents.

putative enzymes may explain the growth on galactan (Table 2). Alternatively the four $\alpha$-arabinofuranosidases (GH51 and GH54) plus the three $\alpha$-L-rhamnosidases (GH78) could be responsible for liberating the other sugars (arabinose, rhamnose) in galactan to support growth.

\section{The preference of Trichoderma virens for lemon pectin} cannot be explained based on its genome content

In contrast to the other species, $T$. virens grew better on lemon pectin than on apple, soy and sugar beet pectin. It also showed good growth on galactan, arabinan and polygalacturonic acid. T. virens has 2 endopolygalacturonases, 2 exopolygalacturonases and 2 exorhamnogalacturonases and $3 \beta$-glucuronyl hydrolases in its genome. These activities are required on substrates rich in uronic acids such as polygalacturonic acid (>97\%), lemon $(88 \%)$, apple $(86 \%)$ and sugar beet $(72.9 \%)$ pectins. The T. virens genome also contains $1 \beta$-galactosidase, $3 \alpha$ rhamnosidases and $2 \alpha$-L-arabinofuranosidases that can be efficiently used to degrade galactan and soy pectin, containing $87 \%$ and $39 \% \mathrm{D}$-galactose, respectively, and potato RGI (20\% L-rhamnose) and arabinan $(88 \% \mathrm{~L}-$ arabinose).

Lemon and apple pectin have a very similar sugar composition. Lemon pectin contains a little less neutral sugars and a little more uronic acids than apple pectin (Table 1). Despite this similarity, G. zeae, B. cinerea, A. nidulans, $P$. anserina and $P$. chysosporium showed somewhat reduced growth on lemon pectin compared to apple pectin. No obvious difference in the CAZyme contents can explain this result. T. virens is the only fungus among the 12 fungi tested in this study that clearly show a preference for lemon pectin.

The poor growth of $T$. virens on arabinan is likely due to the absence of GH43 endoarabinanases, as several other enzymes involved in arabinan degradation were found in the $T$. virens genome. These are two $\alpha$ arabinofuranosidases (GH54), and one exo-arabinanase (GH93). R. oryzae also has a small arabinan-degrading potential with no GH51, GH54 or GH93 members, and only 2 arabinan-related GH43 members. The growth 


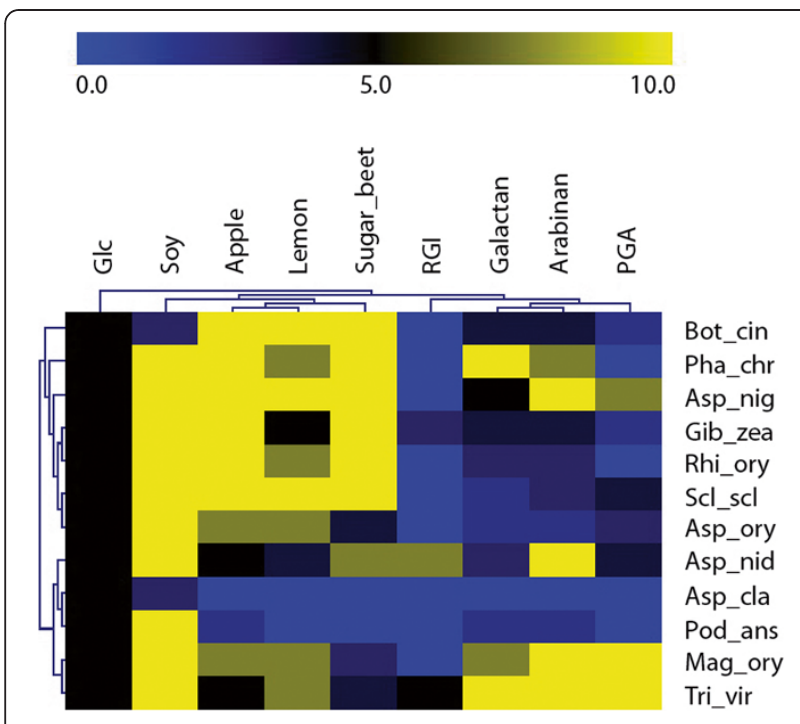

Figure 3 Hierarchical clustering of the fungal species grown on pectins and pectic elements. The growth of the 12 fungal species on the 4 pectins and 4 structural elements was used to generate a distance tree (see material and method for detail on measurements of the growth). The scores are represented by a colour scale from poor/no growth, deep blue to very good growth, yellow. The figure was edited using Mev [57].

profile of these two fungi on arabinan is less good than the growth profile of the other species that are richer in arabinan degrading enzymes such as $A$. niger, $A$. nidulans and $M$. oryzae.

\section{Phanerochaete chrysosporium may use PGA rather than PME to degrade high methyl esterified pectin}

$P$. chrysosporium is poorly equipped with pectin degrading enzymes. Its genome contains only one candidate endopolygalacturonase, one exopolygalacturonase and one endorhamnogalacturonase in $\mathrm{GH} 28$, but does contain three GH35, two GH51, one GH53 and one GH88 members. Although it does not contain pectin methyl esterase it grew well on substrates that have a high methyl esterification degree such as soy pectin (DM 68) and similar but slightly less on apple (DM 72) and lemon pectin (DM 70). Two explanations are proposed. First, the endopolygalacturonase of $P$. chrysosporium may be more active on high methyl esterified pectin, which is supported by the clustering of this gene with $A$. niger PgaA and PgaB in the phylogenic tree (Additional file 3). PgaA and PgaB prefer pectins with a high methyl esterification degree [32]. Secondly, among the pectin structural elements, $P$. chrysosporium prefers galactan and arabinan. The endo-1,4- $\beta$-galactanase (GH53) and the $\beta$-galactosidase $(\mathrm{GH} 35)$ release galactose from galactan, while the $\alpha$-arabinofuranosidase (GH51) releases arabinose from arabinan. As soy pectin contains more galactose and arabinose than the other pectins, this may explain why growth on this pectin is somewhat better than on the other pectins.

Exoarabinanase may be essential for rhamnogalacturonan I degradation to support fungal growth

Several pectinases can act on RGI. Exo- and endorhamnogalacturonases (GH28) and rhamnogalacturonan lyases (PL4) cleave the $\alpha$-1-4-glycosidic bonds between L-rhamnose and D-galacturonic acids of the backbone. $\alpha$-L-rhamnosidase (GH78) release the terminal non-reducing $\alpha$-L-rhamnose residues of RGI, while $\alpha$-L-arabinofuranosidases (GH51, GH54), endo- and exo-arabinanases (GH43, GH93), $\beta$-galactanases and $\beta$-galactosidases (GH53, GH35) act on the side chains.

Interestingly, the growth profiles demonstrate that the species able to grow on RGI all contain one or more genes encoding an exo-arabinanase (GH93) in their genome. This enzyme releases $\alpha-1,5$-L-arabinobiose from the non-reducing end of RGI and is also active on linear L-arabinose oligomers. The reason for fungi to have more than one exo-arabinanase may lay in differences in the substrate specificity of these iso-enzymes. Having multiple slightly different enzymes for the same substrate will ensure efficient hydrolysis and a better utilization of the substrate. $P$ chrysosporium and $R$. oryzae have no GH93. A. clavatus and S. sclerotiorum have one putative GH93 each but the sequences do not contain a signal sequence and the encoded enzymes are therefore likely not secreted. Those species cannot grow on RGI (Figure 4). Few studies have been reported on this enzymatic activity. The structure of the exoarabinanase of G. zeae was recently described and a catalytic mechanism was proposed [34]. This activity is also described in two other fungal species, Penicillium chrysogenum [35], and Chrysosporium lucknowense [36], but they did not address its importance for RGI degradation.

\section{Conclusions}

The data from our study demonstrates that overall there is a clear correlation between the number of genes related to pectin degradation in fungal genomes and the ability of these fungi to grow on pectin. This correlation is less clear when the presence of specific pectin-acting enzymes is compared to growth on pectin structural elements. This indicates that different strategies can be employed by fungi to degrade these structural elements. While it is clear that a relative increase in pectin-related genes in general leads to better growth on pectin, the number of genes per enzyme activity does not necessarily correlate to improved growth on the part of pectin this activity is aimed at.

The differences in phenotype cannot be fully explained by genome content and regulation of the expression of 

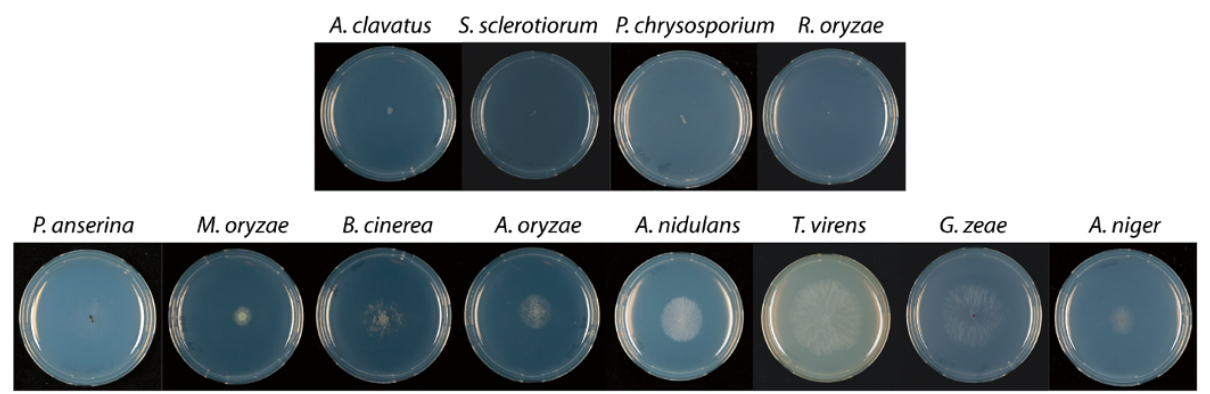

Figure 4 Growth on RGI related to GH93 presence.

the genes is certainly playing an important role. However, fungal regulatory systems of pectinolytic genes are far from fully elucidated. Galacturonic acid is considered to be the main inducer of pectinolytic genes, but other sugars (e.g. arabinose, rhamnose and galactose) are also involved in the induction of subsets of pectinolytic genes in A. niger [37]. So far only the arabinose-responsive regulator (AraR) has been identified [38]. This regulator is only present in the Aspergilli of this study, but not in the other species, which demonstrates that differences in pectin degradation between the species also exist at the regulatory level. Wubben et al. [39] postulated that $B$. cinerea endopolygalacturonases genes are regulated by four systems: basal expression, induction by pectic monomers, glucose repression and modulation by $\mathrm{pH}$. Whether all four systems also affect pectin degradation in the other species is unclear at this point.

Alternatively, the currently available data on these enzymes is insufficient to understand the combined capacity to degrade different structural elements of pectin. One also has to keep in mind that approximately $40 \%$ of the genes of fungal genomes are not associated with a known function or precise substrate specificity [40]. In consequence it is possible that families not yet discovered intervene in the breakdown of specific pectin structural elements. Two recent examples are (i) the identification and characterization of the $\alpha$-glucuronidase from $S$. commune that was assigned to a novel glycoside hydrolase family (GH115) [41] and (ii) the GH127 family created after Fujita et al. [42] who characterized a novel $\beta$-L-arabinofuranosidase from Bifidobacterium longum. In addition, activities currently suggested for members of established CAZy families may be incorrect due to lack of biochemical support, changing the overall comparison of the fungal species.

From the four pectins tested, soy and sugar beet pectin are most different in their composition. Proteomics on the secretome of the 12 fungi grown on these pectins would give an overview of which enzymes are responsible for breaking down these different pectin structures. Future studies could also include other pectins with different properties, such as hop pectin which has a lower degree of methyl esterification than apple and citrus pectin and a relatively high degree of acetylation and neutral sugar content.

This study demonstrates the large possibilities that fungal species offer to efficiently degrade different types of pectin. Pectic structures are extremely diverse, depending on the origin of the plant and on the plant tissue. Fungi evolved in specific biotopes and developed tools adapted to colonize broad range of substrates such as pectins. Interesting points were also observed at the structural element level. In particular, strong indications were found for an important role of exo-arabinanase in RGI degradation.

Our data is a first step to being able to select fungi, which produce highly specific pectinase mixtures. These could be aimed at the production of desired polymers and oligosaccharides, such as those with a positive effect on human health or better colloid properties. In addition, the differences observed between the species can also be used to identify pectinolytic genes that are missing or insufficiently expressed in the current industrial pectinases producers.

\section{Methods}

\section{Chemicals and media}

Aspergillus minimal medium was described by de Vries et al. [43]. Culture conditions and minimum media for $P$. anserina, S. sclerotinia and T. virens were used as respectively described by Silar [44] Cruickshank [45] and Deane et al. [46]. 1\% of the carbon sources (except glucose, see below) were added to the minimal medium containing 1.5\% agarose (Merck,101614) For glucose, $25 \mathrm{mM}$ was used as final concentration. The $\mathrm{pH}$ of the medium was adjusted to 6.0 and autoclaved.

Soy pectin (soybean soluble polysaccharide (SSPS; Soyafibe-S-DA100) was obtained from Fuji Oil Co. Ltd (Osaka, Japan) and prepared from the residue of soy 
Table 4 Culture conditions

\begin{tabular}{lccccc}
\hline \multicolumn{1}{c}{ Species } & Strain number & Reference for genome & Medium & Inoculation & Growth T ( $\left.{ }^{\circ} \mathbf{C}\right)$ \\
\hline Sclerotinia sclerotiorum & ATCC18683 & {$[60]$} & MM Sclerotinia & Mycelial plug & 25 \\
Botryotinia fuckeliana & B05.10 & {$[60]$} & MM Aspergillus & $10^{3} \mathrm{sp}$ & 25 \\
Aspergillus nidulans & FGSC A4 & {$[61]$} & MM Aspergillus & $10^{3} \mathrm{sp}$ & 30 \\
Aspergillus clavatus & NRRL1 & www.broadinstitute.org & MM Aspergillus & $10^{3} \mathrm{sp}$ & 30 \\
Aspergillus oryzae & RIB40 & {$[62]$} & MM Aspergillus & $10^{3} \mathrm{sp}$ & 30 \\
Aspergillus niger & N402 & {$[63]$} & MM Aspergillus & $10^{3} \mathrm{sp}$ & 30 \\
Magnaporthe oryzae & $70-15$ & {$[64]$} & MM Aspergillus & $10^{3} \mathrm{sp}$ & 30 \\
Podospora anserina & S mat+ & {$[65]$} & MM Podospora & Mycelial plug & 30 \\
Trichoderma virens & Gv29-8 & genome.jgi-psf.org & MM Trichoderma & $10^{3} \mathrm{sp}$ & 30 \\
Gibberella zeae & FG05491.1 & {$[66]$} & MM Aspergillus & Mycelial plug & 30 \\
Phanerochaete chrysosporium & RP78 & {$[67]$} & MM Aspergillus & Mycelial plug & 25 \\
Rhizopus oryzae & $99-880$ & MM Aspergillus & $10^{3} \mathrm{sp}$ & 25 \\
\hline
\end{tabular}

protein extraction. Apple and lemon pectin were kindly provided by Degussa Texturant Systems (Baupte, France). Sugar beet pectin was obtained from Copenhagen pectin A/S (Lille Skensved, Denmark). Potato Galactan, sugar beet Arabinan, potato Rhamnogalacturonan I were obtained from Megazyme (Bray, Ireland)., Citrus polygalacturonic acid and glucose were obtained from SigmaAldrich

\section{Strains and growth conditions}

The fungal strains and culture conditions are listed in Table 4. For $A$. niger the commonly used lab strain $\mathrm{N} 402$ [47] was used and compared to the genome of the strain sequenced by JGI which is highly similar. The centre of the plates was inoculated with $2 \mu \mathrm{l}$ of a suspension of 500 spores/ $\mu \mathrm{l}$ or with a small agar plug containing mycelium (1 mm diameter) transferred from the edge of a vigorously growing 3-days-old colony. The cultures were incubated at $25^{\circ} \mathrm{C}$ or $30^{\circ} \mathrm{C}$ as indicated in Table 4. Mycelium density and colony diameter were measured daily. Colony morphology pictures were taken after 3 or 5 days depending on the growth rate of the fungi. The growth test was performed in triplicate for each strain.

\section{Growth evaluation}

Growth was independently scored by two persons. The mycelium density was evaluated by visual inspection of the colonies and combined with the diameter to obtain a score between 1 and 10. Glucose was taken as the internal reference and scored as 5 . A better growth compared to the reference was scored between 6 and 10 when a poor growth was scored between 0 (no growth) and 4. Sporulation was taken in account as a positive criterion of growth.

\section{Sugar composition determination}

Sugar composition was determined after Saeman Hydrolysis. After a prehydrolysis step using $72 \% \mathrm{w} / \mathrm{w}$ sulphuric acid at $30^{\circ} \mathrm{C}$ for $1 \mathrm{~h}$ the samples were hydrolysed with $1 \mathrm{M}$ sulphuric acid at $100^{\circ} \mathrm{C}$ for $3 \mathrm{~h}$. Afterwards the sugars were derivatised as alditol acetates and determined by gas chromatography [48] using inositol as internal standard.

\section{Uronic acid content}

The total uronic acid content was determined with the automated m-hydroxydiphenyl assay [49].

\section{Degree of acetylation and methyl esterification}

Degree of acetylation and methyl esterification were determined by HPLC after hydrolysis with $0.4 \mathrm{~N}$ sodium hydroxide in isopropanol/water (50/50 v/v) [50]. The degree of acetylation and methyl esterification are calculated as mole methyl/acetyl groups per 100 mole galacturonic acid. One mole galacturonic acid can carry only one mol methyl esters and two moles acetyl ester.

\section{CAZy annotation}

The identification step of CAZymes followed the procedures previously described [29] where sequences are subject to BlastP analysis [51] against a library composed of modules derived from the CAZy database. The positive hits are then subjected to a modular annotation procedure that maps the individual modules against libraries of catalytic and carbohydrate models derived from CAZy using BlastP or Hidden Markov models $[52,53]$. The results are augmented with signal peptide, transmembrane, and GPI predictions by human curators [54-56]. The fragmentary models and all models suspected of splicing prediction errors are identified. The functional annotation step involves BlastP comparisons 
against a library modules derived from biochemically characterized enzymes [29].

\section{Hierarchical clustering of pectin degradation capacity and phylogenetic analysis}

To visualize the growth profile of the 12 fungal species on the 4 pectins and 4 structural elements, a Pearson correlation matrix was generated by using $\mathrm{MeV}$, Multiexperiment Viewer [57].

For the construction of the phylogenetic trees of the $\mathrm{GH}$ families the sequences of the family members were aligned using Muscle (version 3.8.31) [58] after which the alignment was used to generate a bootstrapped minimal evolution tree (1000 bootstraps) using MEGA4 [59].

\section{Additional files}

Additional file 1: Phylogeny of the GH28 family.

Additional file 2: Phylogeny of the GH43 family.

Additional file 3: Growth profiles of the 12 fungi on the 4 pectins, the 4 structural elements and glucose.

\section{Competing interests}

The authors declare that they have no competing interests.

\section{Authors' contributions}

IB performed the experiments, analyzed the data and drafted the manuscript. PMC and BH performed comparative genomic analysis. HAS supplied part of the experimental material. RPdV and IB designed the study. All authors contributed to the interpretation of the data. All authors read and approved the final version of the paper.

\section{Acknowledgements}

IB was supported by a grant of the Dutch Technology Foundation STW, Applied Science division of NWO and the Technology Program of the Ministry of Economic Affairs UGC 07938 to RPdV.

\section{Author details}

'Microbiology \& Kluyver Centre for Genomics of Industrial Fermentations, Utrecht University, Padualaan 8, Utrecht 3584 CH, The Netherlands.

${ }^{2}$ Architecture et Fonction des Macromolécules Biologiques, Aix-Marseille Université, CNRS UMR 7257, Case 932, 163 Av de Luminy, Marseille cedex 9 13288, France. 'Laboratory of Food Chemistry, Wageningen University, Bomenweg 2, Wageningen 6703HD, The Netherlands. ${ }^{4}$ Fungal Physiology, CBS-KNAW, Uppsalalaan 8, Utrecht 3584 CT, The Netherlands.

Received: 19 March 2012 Accepted: 7 July 2012

Published: 19 July 2012

\section{References}

1. Mohnen D: Biosynthesis of pectins, In G.B. Seymour, J. P. Knox. Pectins and their manipulation. Oxford: Blackwell Publishing, Ltd; 2002:52-68.

2. Ridley BL, O'Neil MA, Mohnen D: Pectins: structure, biosynthesis, and oligogalacturonide-related signaling. Phytochem 2001, 57:929-967.

3. O'Neill M, Albersheim P, Darvill A: The pectic polysaccharides in primary cell walls, In P. M. Dey. Methods in Plant Biochemistry. London: Academic; 1990:415-441.

4. O'Neill MA, Ishii T, Albersheim P, Darvill AG: Rhamnogalacturonan II: Structure and Function of a Borate Cross-Linked Cell Wall Pectic Polysaccharide. Ann Rev Plant Biol 2004, 55(1):109-139.

5. Visser J, Voragen AGJ: Progress in Biotechnology 14: Pectins and Pectinases. Amsterdam: Elsevier; 1996.

6. Hilz $\mathrm{H}$ : Characterisation of cell wall polysaccharides in bilberries and black currants, PhD thesis. The Netherlands: Wageningen University; 2007. ISBN 90-8504-624-6.
7. Vincken JP, Schols HA, Oomen RJFJ, McCann MC, Ulvskov P, Voragen AGJ, et al: If homogalacturonan were a side chain of rhamnogalacturonan I. Implications for cell wall architecture. Plant Physio/ 2003, 132(4): 1781-1789.

8. Coenen GJ, Bakx EJ, Verhoef RP, Schols HA, Voragen AGJ: Identification of the connecting linkage between homo- or xylogalacturonan and rhamnogalacturonan type I. Carbohyd Polym 2007, 70(2):224-235.

9. Nunez A, Fishman ML, Fortis LL, Cooke PH, Hotchkiss AT: Identification of extensin protein associated with sugar beet pectin. J Agric Food Chem 2009, 57(22):10951-10958.

10. Immerzeel P: Characterisation of carrot arabinogalactan proteins, $\mathrm{PhD}$ thesis. The Netherlands: Wageningen University; 2005. ISBN 90-8504-150-3.

11. Drusch S: Sugar beet pectin: A novel emulsifying wall component for microencapsulation of lipophilic food ingredients by spray-drying. Food Hydrocolloids 2007, 21:1223-1228.

12. Kastner U, Glasl S, Follrich B, Guggenbichler JP, Jurenitsch J: Acid oligosaccharides as the active principle of aqueous carrot extracts for prevention and therapy of gastrointestinal infections. Wien Med Wochenschr 2002, 152:379-381.

13. Trumbo $P$, Schlicker $S$, Yates AA, et al: Dietary reference intakes for energy, carbohydrate, fiber, fat, fatty acids, cholesterol, protein and amino acids. J Am Diet Assoc 2002, 102:1621-1630.

14. Glinsky W, Raz A: Modified citrus pectin anti-metastasic properties: one bullet, multiple targets. Carbohyd Res 2009, 344:1788-1791.

15. Nangia-Makker $\mathrm{P}$, Hogan $\mathrm{V}$, Honjo $\mathrm{Y}$, Baccarini $\mathrm{S}$, Tait $L$, Bresalier $R$, Raz A: Inhibition of human cancer cell growth and metastasis in nude mice by oral intake of modified citrus pectin. J Natl Cancer Inst 2002, 94(24):1854-1862.

16. Olano-Martin E, Gibson GR, Rastall RA: Comparison of the in vitro bifidogenic properties of pectins and pectic-oligosaccharides. J App/ Microb 2002, 93:505-511.

17. Holck J, Hjernø K, Lorentzen A, Vigsnæs LK, Hemmingsen L, Mikkelsen TR $L J$, Meyer AS: Tailored enzymatic production of oligosaccharides from sugar beet pectin andevidence of differential effects of a single DP chain length difference on human faecal microbiota composition after in vitro fermentation. Process Biochem 2011, 46:1039-1049.

18. Schols HA, Voragen AGJ: Complex pectins: Structure elucidation using enzymes. In Progress in Biotechnolology, Pectins and pectinases, Volume 14. Edited by Visser J, Voragen AGJ. Amsterdam, Netherlands: Elsevier Science B. V; 1996:3-19.

19. Benen JAE, Vincken JP, van Alebeek GJWM: Microbial pectinases, In G.B. Seymour, J. P. Knox. Pectins and their manipulation. Oxford: Blackwell Publishing, Ltd; 2002:174-221.

20. Battaglia E, Benoit I, van den Brink J, Wiebenga A, Coutinho PM, Henrissat B, de Vries RP: Carbohydrate-active enzymes from the zygomycete fungus Rhizopus oryzae: a highly specialized approach to carbohydrate degradation depicted at genome level. BMC Genomics 2011, 12:38.

21. Xiong $X$, Wen $X$, Bai $Y$, Qian $Y$ : Effects of culture conditions on ligninolytic enzymes and protease production by Phanerochaete chrysosporium in air. J Environm SC 2008, 20:94-100.

22. Longacre A, Reimers JM, Gannon JE, Wright BE: Flux analysis of glucose metabolism in Rhizopus oryzae for the purpose of increasing lactate yields. Fung Genet Biol 1997, 21:30-39.

23. Saito K, Kawamura Y, Oda Y: Role of the pectinolytic enzyme in the lactic acid fermentation of potato pulp by Rhizopus oryzae. J Ind Microbiol Biotechnol 2003, 30:440-444.

24. Mertens JA, Burdick RC, Rooney AP: Identification, biochemical characterization, and evolution of the Rhizopus oryzae 99-880 polygalacturonase gene family. Fung Genet Biol 2008, 45:1616-1624.

25. Clardy J, Springer JP, Büchi G, Matsuo K, Wrightman R: Tryptoquivaline and tryptoquivalone, two new tremorgenic metabolites of Aspergillus clavatus. J Am Chem Soc 1975, 97:663-665.

26. Buchi G, Kin Chun L, Kobbe B: Townsend JM: Four new mycotoxins of A. clavatus related to tryptoquivaline. J Org Chem 1977, 42:244-246.

27. Varga J, Rigó K, Tóth B, Téren J, Kozakiewicz Z: Evolutionary relationships among Aspergillus species producing economically important mycotoxins. Food Technol Biotechnol 2003, 41:29-36.

28. Cantarel BL, Coutinho PM, Rancurel C, Bernard T, Lombard V, Henrissat B: The Carbohydrate-Active EnZymes database (CAZy): an expert resource for Glycogenomics. Nucleic Acids Res 2009, 37(Database issue): D233-D238. 
29. Coutinho PM, Andersen MR, Kolenova K, VanKuyk PA, Benoit I, Gruben BS, Trejo-Aguilar B, Visser H, Van Solingen P, Pakula T, Seiboth B, Battaglia E, Aguilar-Osorio G, De Jong JF, Ohm RA, Aguilar M, Henrissat B, Nielsen J, Stålbrand H, De Vries RP: Post-genomic insights into the plant polysaccharide degradation potential of Aspergillus nidulans and comparison to Aspergillus niger and Aspergillus oryzae. Fung Genet Biol 2009, 46:S161-S169.

30. Espagne E, Lespinet O, Malagnac F, Da Silva C, Jaillon O, Porcel BM, Couloux A, Aury JM, Ségurens B, Poulain J, Anthouard V, Grossetete S, Khalili H, Coppin E, Déquard-Chablat M, Picard M, Contamine V, Arnaise S, Bourdais A, Berteaux-Lecellier V, Gautheret D, de Vries RP, Battaglia E, Coutinho PM, Danchin EGJ, Henrissat B, EL Khoury R, Sainsard-Chanet A, Boivin A, PinanLucarré $B$, Sellem $C H$, Debuchy $R$, Wincker $P$, Weissenbach J, Silar P: The genome sequence of the model ascomycete fungus Podospora anserina. Genome Biol 2008, 9(5):R77.

31. Martinez D, Berka RM, et al: Genome sequencing and analysis of the biomass-degrading fungus Trichoderma reesei (syn. Hypocrea jecorina) Nature Biotech 2008, 26:553-560.

32. Parenicová $L$, Benen JA, Kester $H C$, Visser J: pgaA and pgaB encode two constitutively expressed endopolygalacturonases of Aspergillus niger. Biochem J 2000, 345:637-644.

33. Mohamed SA, Christensen TMIE, Mikkelsen JD: New polygalacturonases from Trichoderma reesei: characterization and their specificities to partially methylated and acetylated pectins. Carbohyd Res 2003, 338:515-524.

34. Carapito R, Imberty A, Jeltsch JM, Byrns SC, Tam PH, Lowary TL, Varrot A, Phalip V: Molecular basis of arabinobio-hydrolase activity in phytopathogenic fungi. J Bio Chem 2009, 284(18):12285-12296.

35. Sakamoto T, Ihara H, Shibano A, Kasai N, Inui H, Kawasaki H: Molecular characterization of a Penicillium chrysogenum exo-1,5-K-L-arabinanase that is structurally distinct from other arabinan-degrading enzymes. FEBS Lett 2004, 560:199-204.

36. Kühnel S, Hinz SWA, Pouvreau L, Wery J, Schols HA, Gruppen H: Chrysosporium lucknowense arabinohydrolases effectively degrade sugar beet arabinan. Bioresour Technol 2010, 101:8300-8307.

37. de Vries RP, Jansena J, Aguilara G, Parenicova L, Joosten V, Wulfert F, Benena JAE, Visser J: Expression profiling of pectinolytic genes from Aspergillus niger. FEBS Lett 2002, 530:41-47.

38. Battaglia E, Visser L, Nijssen A, van Veluw GJ, Wösten HAB, de Vries RP: Analysis of regulation of pentose utilisation in Aspergillus niger reveals evolutionary adaptations in Eurotiales. Studies in Mycology 2011, 69:31-38.

39. Wubben JP, ten Have A, van Kan JAL, Visser J: Regulation of endopolygalacturonase gene expression in Botrytis cinerea by galacturonic acid, ambient $\mathrm{pH}$ and carbon catabolite repression. Curr Genet 2000, 37:152-157.

40. Galagan JE, Henn MR, Ma L, et al: Genomics of the fungal kingdom: Insights into eukaryotic biology. Genome Res 2005, 15:1620-1631.

41. Chong S-L, Battaglia E, Couthino PM, Henrissat B, Tenkanen M, De Vries RP: The a-glucuronidase Agu1 from Schizophyllum commune is a member of a novel glycoside hydrolase family (GH115). Appl Microbiol Biotechnol 2011, 90:1323-1332.

42. Fujita K, Takashi Y, Obuchi E, Kitahara K, Suganuma T: Characterization of a Novel $\beta$-L-Arabinofuranosidase in Bifidobacterium longum: a functional elucidation of a DUF1680 family member. J Bio Chem 2011, 286 (44):38079-38085.

43. De Vries RP, Burgers K, van de Vondervoort PJI, Frisvad JC, Samson RA Visser J: A new black Aspergillus species, A. vadensis, is a promising host for homologous and heterologous protein production. Appl Environ Microbiol 2004, 70:3954-3959.

44. Silar P: Grafting as a method for studying development in the filamentous fungus Podospora anserina. Fungal Biol 2011, 115:793-802.

45. Cruickshank RH: Distinction between Sclerotinia species by their pectic zymograms. Trans. Brit. Mycol. Soc. 1983, 80:117-119.

46. Deane EE, Whipps JM, Lynch JM, Peberdy JF: The purification and characterization of a Trichoderma harzanium exochitinase. Biochim Biophys Act 1998, 1383:101-110.

47. Bos CJ, Debets AJM, Swart K, Huybers A, Kobus G, Slakhorst SM: Genetic analysis and the construction of master strains for assignment of genes to six linkage groups in Aspergillus niger. Current Genet 1988, 14:437-443.
48. Englyst HN, Cummings $\mathrm{JH}$ : Simplified method for the measurement of total non-starch polysaccharides by gas-liquid chromatography of constituent sugars as alditol acetates. Analyst 1984, 109(7):937-942.

49. Thibault JF: Automatisation du dosage des substances pectiques par la methode au meta-hydroxydiphenyl. Lebensmittel Wissenschaft und Technologie Food Science and Technology 1979, 12(5):247-251.

50. Voragen AGJ, Schols HA, Pilnik W: Determination of the degree of methylation and acetylation of pectins by h.p.l.c. Food Hydrocolloids 1986, 1(1):65-70.

51. Altschul SF, Madden TL, Schaffer AA, Zhang J, Zhang Z, Miller W, Lipman DJ: Gapped BLAST and PSI-BLAST: a new generation of protein database search programs. Nucleic Acids Res 1997, 25(17):3389-3402.

52. Eddy SR: Profile hidden Markov models. Bioinformatics 1998, 14(9):755-763.

53. Eisenhaber B, Schneider G, Wildpaner M, Eisenhaber F: A sensitive predictor for potential GPI lipid modification sites in fungal protein sequences and its application to genome-wide studies for Aspergillus nidulans, Candida albicans, Neurospora crassa, Saccharomyces cerevisiae and Schizosaccharomyces pombe. J Mol Biol 2004, 337(2):243-253.

54. Fankhauser N, Maser P: Identification of GPI anchor attachment signals by a Kohonen self-organizing map. Bioinformatics 2005, 21(9):1846-1852.

55. Pierleon A, Martelli PL, Casadio R: PredGPI: a GPI-anchor predictor. BMC Bioinformatics 2008, 9:392.

56. Kall L, Krogh A, Sonnhammer EL: A combined transmembrane topology and signal peptide prediction method. J Mol Biol 2004, 338(5):1027-1036.

57. Saeed Al, Sharov V, White J, Li J, Liang W, Bhagabati N, et al: TM4: a free, open-source system for microarray data management and analysis. Biotechniques 2003, 34(2):374-378.

58. Edgar RC: MUSCLE: multiple sequence alignment with high accuracy and high throughput. Nucleic Acids Res 2004, 32(5):1792-1797.

59. Tamura K, Dudley J, Nei M, Kumar S: MEGA4: Molecular Evolutionary Genetics Analysis (MEGA) software version 4.0. Mol Bio Evol 2007, 24:1596-1599.

60. Amselem J, Cuomo CA, van Kan JAL, Viaud M, Benito EP, et al: Genomic Analysis of the Necrotrophic Fungal Pathogens Sclerotinia sclerotiorum and Botrytis cinerea. PLoS Genet 2011, 7(8):e1002230. doi:doi:10.1371/ journal.pgen.1002230.

61. Wortman JR, Gilsenan JM, Joardar V, Deegan J, Clutterbuck J, Andersen MR, Archer D, Bencina M, Braus G, Coutinho P: The 2008 update of the $A$. nidulans genome annotation: A community effort. Fung Genet Biol 2009, 46(1):S2-S13.

62. Machida M, Asai K, Sano M, Tanaka T, Kumagai T, Terai G, Kusumoto K, et al: Genome sequencing and analysis of Aspergillus oryzae. Nature 2005, 438:1157-1161.

63. Andersen MR, Salazar MP, Schaap PJ, et al: Comparative genomics of citricacid-producing Aspergillus niger ATCC 1015 versus enzyme-producing CBS 513.88. Genome Res 2011, 21:885-897.

64. Dean RA, Talbot NJ, Ebbole DJ, Farman ML, Mitchell TK, et al: The genome sequence of the rice blast fungus Magnaporthe grisea. Nature 2005, 434:980-986.

65. Espagne E, Lespinet O, Malagnac F, Da Silva C, Jaillon O, Porcel BM, Couloux A, Aury JM, Ségurens B, Poulain J, Anthouard V, Grossetete S, Khalili H, Coppin E, Déquard-Chablat M, Picard M, Contamine V, Arnaise S, Bourdais A, Berteaux-Lecellier V, Gautheret D, de Vries RP, Battaglia E, Coutinho PM, Danchin EGJ, Henrissat B, Khoury REL, Sainsard-Chanet A, Boivin A, PinanLucarré $B$, et al: The genome sequence of the model ascomycete fungus Podospora anserina. Genome Biol 2008, 9:R77.

66. Ma LJ, van der Does HC, Borkovich KA, Coleman JJ, Daboussi MJ, Di Pietro A, Dufresne M, Freitag M, Grabherr M, Henrissat B, et al: Comparative genomics reveals mobile pathogenicity chromosomes in Fusarium. Nature 2010, 464:367-373.

67. Martinez D, Larrondo LF, Putnam N, Gelpke MDS, Huang K, et al: Genome sequence of the lignocellulose degrading fungus Phanerochaete chrysosporium strain RP78. Nature Biotech 2004, 22:695-700.

68. Ma $\sqcup$, Ibrahim AS, Skory C, Grabherr MG, Burger G, Butler M, Elias M, Idnurm A, Lang BF, Sone T, et al: Genomic analysis of the basal lineage fungus Rhizopus oryzae reveals a whole-genome duplication. PLOS Genet 2009, 5(7):e1000549.

doi:10.1186/1471-2164-13-321

Cite this article as: Benoit et al:: Degradation of different pectins by fungi: correlations and contrasts between the pectinolytic enzyme sets identified in genomes and the growth on pectins of different origin. BMC Genomics 2012 13:321. 